\title{
Trade Liberalization, Urbanization and Nutrition Transition in Asian Countries
}

\author{
Ghose Bishwajit $^{1 *}$, Seydou Ide ${ }^{2}$, Mohammad Akmal Hossain ${ }^{3}$ and Md Nurus Safa \\ ${ }^{1}$ Institute of Nutrition and Food Science, University of Dhaka, Bangladesh \\ ${ }^{2}$ Faculty of Health Sciences, University of Ottawa, Canada \\ ${ }^{3}$ School of Public Health, Tongji Medical College, China \\ ${ }^{4}$ Huazhong Normal University, School of Journalism and Communication, Wuhan University of Technology, China
}

Received: December 12, 2013; Accepted: January 27, 2014; Published: January 29, 2014

*Corresponding author: Ghose Bishwajit, Institute of Nutrition and Food Science, University of Dhaka, Bangladesh, E-mail: brammaputram@ gmail.com

\begin{abstract}
Objective: This paper describes few crucial determinants of nutrition transition in Asia such as liberalization of agrifood trade and urbanization. It also shows that the nutrition transition is concurrent with an epidemiological transition and proposes strategies to control the adverse effects of these events which have huge implications for the social and economic development.

Design: This article reviews the already published literature on nutrition and epidemiological transition. Pubmed and Medline database were searched for articles published between 1985 to 2012 by using the terms of nutrition transition, impact of trade liberalization on health, urbanization in Asia, dietary transition in Asian countries, epidemiological transition in Asia. Research papers on nutrition transition in few non-Asian countries were also reviewed to show their policies on obesity, public health and nutrition.

Conclusion: Asian countries are undergoing a rapid nutritional and epidemiological transition which has a huge impact on the rising prevalence of chronic non-communicable diseases like diabetes, obesity and other associated diseases. Policymakers should focus on formulating strategies to curb the incidence of these costly diseases at both community and national level to address the economic as well as the social implications of these diseases.
\end{abstract}

Keywords: Nutrition transition; Epidemiological transition; Non-communicable diseases; Trade liberalization; Urbanization; Asia

\section{Abbreviations}

DR-NCDs: Diet-Related Non-Communicable Diseases; IPTFA: Industrially Produced Trans Fatty Acids; WTO: World Trade Organization

\section{Introduction}

The nutritional transition currently occurring in Asia is a combined result of demographic, nutritional, and epidemiological transition accompanied by rapid urbanization and economic growth at an unprecedented rate [1,2]. Over the past two decades, there has been increasing evidence that the structure of dietary intakes and the prevalence of diet-related non-communicable diseases (DR-NCDs) around the developing world have been undergoing considerable shifts. India is reported to be facing an epidemic of diet-related non-communicable diseases [3]. The nutrition transition model was first proposed by Popkin BM [1], and since then the subject has drawn attention to nutritionists and heath specialists from many countries $[4,5]$. Directed by the advancement in agriculture, modern food science and technology and globalization of food system, the type and quantity of food consumed have changed dramatically both in developed and developing societies [6]. Asian countries likewise have witnessed considerable nutritional, demographic and epidemiological changes over past few decades marked by lower infant mortality rate, increased life expectancy, higher incidences of chronic noncommunicable diseases (NCDs) [7]. The nutrition transition, in general, is characterized by a shift from a high proportion of locally grown staple grains, legumes, low animal based food, toward more varied diets comprising more refined, energydense, animal based and processed food which are generally high in sugar and fat and low in carbohydrate content. This shift in dietary habit, accompanied by reduced physical activity and leisure period lead to increase in overweight and obesity over time [8]. Once known as 'the diseases of affluence', developing countries in Asia such India, Bangladesh, Nepal is now facing a disproportionate burden of NCDs and an increasing number of households are affected concomitantly under nutrition and obesity [9]. Obesity and obesity induced diseases are gradually clustering among the poor communities in Asia and the number of overweight people has already outgrown that of undernourished people in some regions.

\section{Impact of trade liberalization and urbanization on nutrition transition}

Though some countries have seen the advantages of open trade regimes, most of the countries (especially the developing ones) are seem to be negatively affected by some highly controversial policies. Trade liberalization is believed to be associated with greater income inequality, unemployment, under employment and has facilitated availability of highly processed 
food in developing countries [10]. Quite evidently, globalization and cross-border trade have spurred economic growth in many Asian countries; but its subsequent social, environmental and health costs are also unignorable, especially with food price volatility, food insecurity and higher incidence of NCDs. Changes in economic structure are generally associated with nutrition transition, and a shift from a labor-intensive preindustrial agrarian economy to machine-intensive industrialized economy causes a similar shift in time allocation and physical and leisure activities [11]. By the advent of modern food technologies (access to cheap edible oils, sugar, food additives) and expansion of fast food and supermarket chain and regulatory environments (WTO) are greatly contributing to nutrition and epidemiological shifts in LMICs (low-and-middle income countries) [12]. Researchers suggest that as nations economic status improves, it also increases the risk of diet related NCDs influenced by factors such as changes in diet pattern, more mechanized lifestyle with less physical activities, urbanization, restructuring of the food system, environmental degradation which are characteristic of industrialized countries. Trade liberalization has brought a rapid economic growth in many countries, economic changes throughout the world which also have prompted a host of nutrition related problems such as decreased prevalence of under nutrition, an increase in the prevalence of diet related diseases and so on [13]. Over the last 50 years, the world has witnessed a dramatic growth of its urban population and at an alarming scale especially in the less developed regions, which continue to pose formidable challenges to the individual countries as well as to the world community [14]. As nutrition transition is driven by forces such as urbanization, industrialization and globalization, it is not surprising that people are gradually adopting western lifestyle with more sedentary type works, limited spaces for physical exercise, which ultimately lead to weight gain. Patterns of obesity from nationally representative surveys indicate that the problems of obesity and dietary excess represent an important challenge facing many lower income countries, particularly their urban populations [15]. Urbanization also fomented the increasing trend of non communicable diseases such as diabetes, cancer, cardiovascular diseases, chronic pulmonary diseases. Urban residents exhibit significant differences in diet, physical activity, mode of living than rural residents. In Thailand, the prevalence of overweight and obesity among children and adolescents has increased considerably during the past 20 years and is more pronounced in children from private schools and urban communities than in those from public schools or rural areas [16].

\section{Epidemiologic transition and the double burden of malnutrition in Asia}

The epidemiological transition, particularly the rapid shift in morbidity and mortality patterns toward much higher rates of non communicable diseases, is dominating the health profile of an increasingly large number of persons in middle- and lowerincome countries [2]. Obesity and associated diseases have become a serious public health issue in Asia and the prevalence tends to increase with level of development and urbanization
[2]. The prevalence of overweight and obesity has increased substantially in the poorer countries, and the economic burden of obesity-related complications is causing farther impoverishment. During the last 30 years obesity has increased worldwide to the extent that those overweight now outnumber those with under nutrition [17]. Countries are undergoing dietary changes with increased meat consumption which together with activity patterns are fueling the obesity epidemic [11]. However, some believe that the distribution of obesity and other NCDs has little to do with meat consumption. It has more to do with the excessive intake of refined sugars and carbohydrates and lack of exercise. However, the combination of a reduction of dietary fat and energy, and increased physical activity, has been shown to reduce the incidence of diabetes by 58 percent in two major trials $[18,19]$. Though obesity is not always correlated with income and diet patterns, it is emphasized that obesity is now affecting the urban poor as well as the rich of Asia. There has been a growing body of research showing that the international standards, used to delineate who is overweight and obese, are not appropriate for many large subpopulations in the world. For instance, a BMI of 25 in an Asian adult appears to have a far greater adverse metabolic effect than in a Caucasian adult [13]. Sedentary lifestyles have been associated with the urban living environment with increased probability of being overweight and obese in India [20]. Researchers also suggest that obesity increases in levels with development and urbanization. Figure 1 shows though burden of NCDs in Asia is lower compared to other developing regions, the prevalence of these diseases is still on the rise. In Asia, the largest number of undernourished people lives in South Asia and the Asia-Pacific region, yet obesity has been recognized as a major disease in this region. However, in South Korea, obesity is low because of high vegetable and low fat intakes. Between 3 and 15 percent of Asian households contain both underweight and overweight individuals, typically an underweight child and an overweight adult [21]. In Sri Lanka in 1995, dietary factors contributed between 10 and 20 percent risks for these chronic diseases and it is projected that NCDs will account for around 21 percent of total death burden in 2025 [21]. In China, diet related chronic diseases accounted for 41.6 percent of all deaths and 22.5 percent of all hospital expenditures

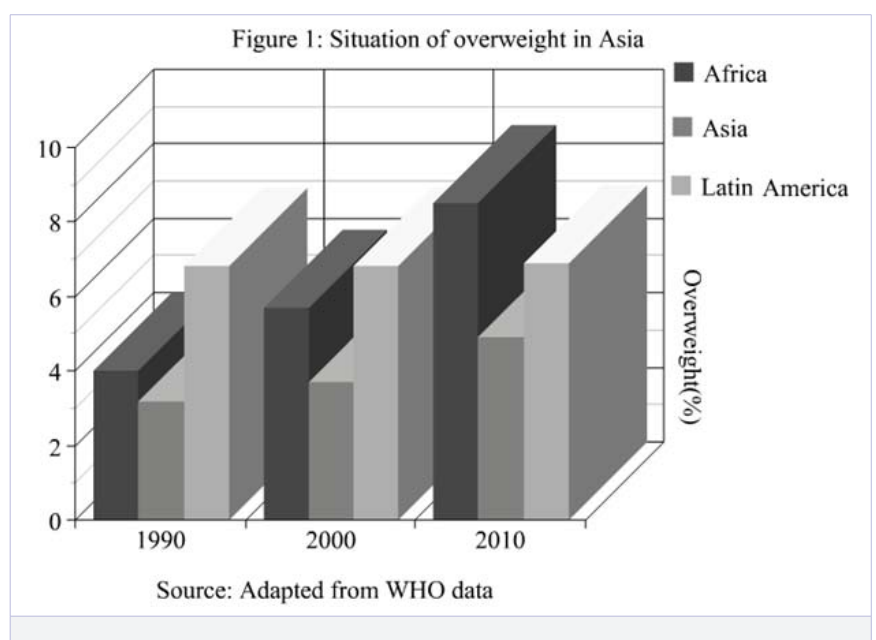


in 1995. The economic costs for these diet related diseases are estimated to be 2.1 percent of annual GDP. Today, diet related NCDs are attributable to a minimum one-fifth to one-quarter of all diseases in China [2]. In Malaysia, cardiovascular diseases (CVD) constitute a major component of diseases of the circulatory system, which increased in prevalence from 7.5 percent in 1965 to 27.8 percent in 1997 . Mortality rates of CVD doubled between 1970 and 1996, from 24.1 to 54.8 per 100000 inhabitants [22]. The prevalence of obesity is comparatively lower in South Korea than in other Asian countries and in countries in the West [23]. In 1998, the obesity rate among adults was 1.7 percent for men and 3.0 percent for women, which was an increase of 0.9 percentage for men and 0.8 percentage for women from 1995, which means an increase of 6.3 percentage points for men and 3.6 percentage points for women in three years [6]. South East Asia has the lowest mean prevalence of overweight women among all world regions, but this may be related to biological differences across ethnicities. Overweight prevalence in adult women ranges from a low of $5 \%$ in Timor-Leste to a high of $29 \%$ in the Philippines.

\section{Nutrition transition in Asia}

Nutrition transition in Asia is marked by a decreasing intake of cereals, vegetables, legumes and an increasing intake of dairy, fish, meat and meat products. Food balance data from the Food and Agriculture Organization (FAO) show that the change in energy intake in Asian countries have been small, but there have been large changes in consumption of animal products, sugars and fats. There appears to be a surge in meat consumption in the Asian countries and animal protein has become increasingly important in Asian diets over the past decade and the consequences for diets, physical activity, and health have been enormous [24]. It is often suggested that the levels of meat consumption correlate with the overall economic development of a given country. In China, per capita meat consumption increased from $9 \mathrm{~kg}$ per capita to more than $50 \mathrm{~kg}$ in the last three decades. Ever since China has introduced reforms in health and economic sectors, a rapid economic growth and continuing increases in income have also brought about important changes to the traditional Chinese diet that resemble to those seen in the western countries [25]. Figure 2 shows that the demand for meat and dairy products has been raising constantly in China since 1980 s. Comparison of food consumption patterns in India also shows a gradual reduction in cereal grain consumption between 1975 and 1995 with a sharp increase in the consumption of animal, milk and milk products, fats and oils [26]. Consumption of pulses and legumes fell sharply during this period. Diets with a higher meat content put additional pressure on land resources for pasture and coarse grain markets for feed, including maize. It is predicted that by 2020, more than 60 percent of meat and milk consumption will occur in the developing world, and the production of beef, meat, poultry, pork and milk will at least double from their 1993 levels [6]. South Korea, one of the most modernized country in Asia, has also been experiencing a rapid transition in diet and nutrition. In the 1980s, new technologies were introduced in South Korea which brought a noticeable expansion in the food-processing industry and lifting restrictions on importation of meat have contributed to increased

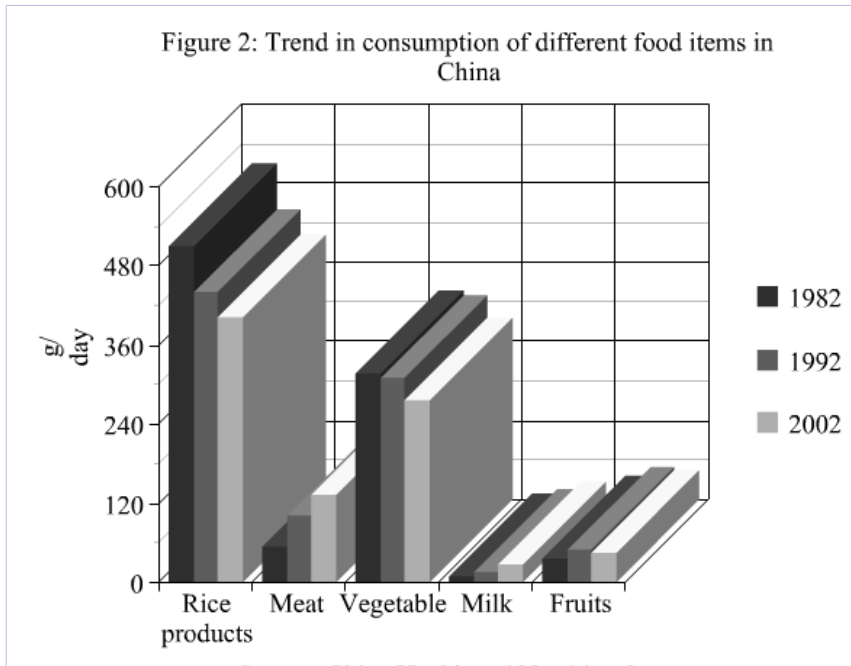

Source: China Health and Nutrition Survey

consumption of all kinds of meat products. Carbohydrate intake has decreased gradually since 1940 from 81 percent of total energy intake to 64 percent in 1995 while intake of meat and poultry increased 10-fold between in the same period [27]. Daily per capita fat intake in South Korea more than doubled from 16.9 $\mathrm{g}$ in 1969 to $41.5 \mathrm{~g}$ in 1998 [27]. However, the predominance of rice as a staple food has helped maintain fat intake relatively lower in Korean diet than in other industrialized nations in Asia. Thailand has been faced with the phenomena of economic and health transitions since the mid 1980s and the economic structure has shifted from agriculture to industry and commerce, resulting in inequitable income distribution. During 1960-1995, the per capita consumption of tubers and cereals, especially rice, declined by about one-third but increasing trend was observed in the consumption of fats, oils, and meat products [16]. Studies show a similar trend in diet in Malaysia characterized by a shift towards an affluent diet that is high in calorie and fat content and low in complex carbohydrate foods [22]. The same study showed by using the FAO food balance sheet (1961 to 1997) that, from 1961 to 1997, the amount of calories obtained from cereals decreased from 61 percent to 41 percent while calories from meat, eggs and fish increased from 6.2 percent to 14.3 percent, sweeteners from 9.5 percent to 18 percent, and oils and fats from 11.4 percent to 14.8 percent respectively. In China, per capita annual consumption of animal foods more than tripled from a very low consumption level of $11 \mathrm{~kg}$ in 1952 to $38 \mathrm{~kg}$ in 1992 and during the period between 1989 and 1997, the total mean intake of cereals decreased by $127 \mathrm{~g}$ per person per day [28].

\section{Challenges for food and nutrition policy}

The existing double burden of malnutrition in Asian countries is exacerbated by the adverse consequences of nutrition transition such as the increasing prevalence of obesity and other associated NCDs [13]. There is an urgent need to shift the relative prices of a range of foods to encourage healthier, less energy dense and more nutrient dense foods along with behavior modification and the promotion of appropriate dietary practices and physical 
exercise. Governments and health workers in developing countries need to adopt comprehensive approaches to tackle the double burden of disease that follows epidemiological and nutritional transition [29]. Policy makers need to make strategies in an attempt to addresses two of the main risk factors for NCDsunhealthy diet and physical inactivity. Dietary habit is becoming increasingly dominated by processed food in the developing countries which has become popular (the product and/or the technology) in the developing countries since the liberalization of global food market. Food pricing strategies have been proposed by many researchers to bring about the shift to healthy eating patterns. Increased taxes on carbonated drinks and saturated fat and subsidies on fruits and vegetables would be associated with beneficial dietary change. Consumers in most Asian countries are very price sensitive, and thus strategic controlling of price can be instrumental in lowering the consumption of unhealthy foods which can be beneficial for minimizing the negative effects of this nutrition transition. Figure 3 shows that meat consumption has increased in all five major economies in South East and Central Asian regions, and controlling meat prices could, to some extent make consumers rely more on vegetable and cereal based foods. It is also now widely believed that increasing international trade alone is unlikely to reduce poverty or food insecurity and its role in global epidemiological and nutrition transitions is also significant. It is therefore crucial in controlling the trade of certain types of food and beverages which are generally considered as obesogenic. Observational studies have found that a high intake of industrially produced trans fatty acids (IP-TFA) increases the risk of weight gain and a gain in abdominal fatness greater than elsewhere in the body. Some European countries, for example in Denmark, introduced legislation restricting the content of IPTFA in all food products to a maximum of 2 percent of their fat content in 2003 [27]. Strategic pricing of edible oil is important to regulate its cheap availability and immoderate consumption which can consequently lead to adverse health effects. Since social and economic determinants have very direct impact on

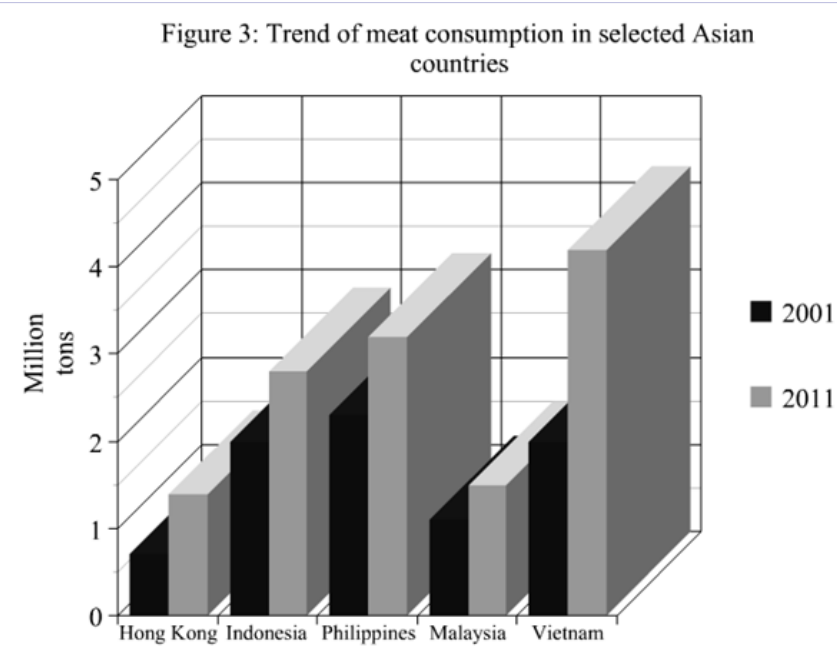

Source: China Health and Nutrition Survey (1989-1997) developing obesity and other NCDs, therefore, besides addressing under nutrition, the nutrition policies in Asian countries also have to focus on these determinants as a strategy for long term prevention of these diseases.

\section{Conclusion}

Asian countries have entered a stage of nutrition led by phenomenal economic growth in past few decades. Rapid urbanization, cross-border trade and advance agrifood technologies have revolutionized Asian food culture marked by considerable decrease in cereal grain and vegetable consumption to high animal based, fat and dairy food consumption. High rate of urbanization has its impacts on people's dietary habit, mode of entertainment, physical activity which together lead to an epidemiological transition where the disease burden is gradually shifting from communicable and infectious diseases to non-communicable chronic diseases. Prevalence of overweight, obesity and other associated diseases like diabetes, cancer, cardiovascular diseases has increased dramatically over past two decades. Apart from high economic cost, these diseases have huge social implications. Asian countries are likely to continue to bear an enormous burden of NCDs, and the economic costs of the total disability-adjusted life years lost are extremely high. A major first step toward a healthier population is awareness of the problems related to the nutrition transition. Asian countries must continue their efforts and develop programs and policies for agricultural production, nutrition, food marketing, and education that will help them to successfully confront the challenges of nutrition transition and achieve sustainable food and nutrition security. National food and agricultural policies, that consider diet-related chronic diseases, are a future priority. Given the current situation of nutritional and epidemiological transition in Asia, an interdisciplinary and concerted approach is required by nutritionists, health policy makers and health sociologists to cope up with the health and social effects of nutrition transition and the double burden of malnutrition in Asian countries.

\section{Acknowledgement}

Authors didn't receive any particular funding for this research.

\section{References}

1. Popkin BM (1993) Nutritional Patterns and Transitions. Population and Development Review 19(1): 138-157.

2. Du S, Lu B, Zhai F, Popkin BM (2002) A new stage of the nutrition transition in China. Public Health Nutr 5(1A): 169-174.

3. Misra A, Singhal N, Sivakumar B, Bhagat N, Jaiswal A, et al. (2011) Nutrition transition in India: secular trends in dietary intake and their relationship to diet-related non-communicable diseases. J Diabetes 3(4): 278-292.

4. Popkin BM, Adair LS, Ng SW (2012) Global nutrition transition and the pandemic of obesity in developing countries. Nutr Rev 70(1): 3-21.

5. Caballero B (2007) The global epidemic of obesity: an overview. Epidemiol Rev 29: 1-5.

6. Hoffman DJ (2010) Early nutrition and adult health: Perspectives for international and community nutrition programs and policies. Nutr 
Res Pract 4(6): 449-454.

7. Escovitz GH (1992) The health transition in developing countries: a role for internists from the developed world. Ann Intern Med 116(6): 499-504.

8. Popkin BM (2001) The Nutrition Transition and Obesity in the Developing World. J Nutr 131(3): 871S-873S.

9. Grijalva-Eternod CS, Wells JCK, Cortina-Borja M, Salse-Ubach N, Tondeur MC, et al. (2012) The Double Burden of Obesity and Malnutrition in a Protracted Emergency Setting: A Cross-Sectional Study of Western Sahara Refugees. PLoS Med 9(10): e1001320.

10. Paula LG, Margaret EB (2001) The nutrition transition is underway in India. J Nutr 131(10): 2692-2700.

11. Almiron-Roig E, Drewnowski A (2003) Hunger, thirst, and energy intakes following consumption of caloric beverages. Physiol Behav 79(4-5): 767-773.

12. Almiron-Roig E, Chen Y, Drewnowski A (2003) Liquid calories and the failure of satiety: how good is the evidence? Obes Rev 4(4): 201-212.

13. Popkin BM, Horton S, Soowon K (2001) The Nutrition Transition and Prevention of Diet-related Diseases in Asia and the Pacific. Food and Nutrition Bulletin 22(4) (supplement).

14. Chamie MJ (2004) World Urbanization Prospects: The 2003 Revision. World Urbanization Prospects, New York, USA, p. 1-3.

15. Popkin BM (1999) Urbanization, lifestyle changes and the nutrition transition. World Development 27(11): 1905-1916.

16. Shetty PS (2002) Nutrition transition in India. Public Health Nutr 5(1A): 175-182.

17.Zhai F, Wang H, Du S, He Y, Wang Z, et al. (2007) Lifespan nutrition and changing socio-economic conditions in China. Asia Pac J Clin Nutr 16(Suppl 1): 374-382.

18. Astrup A, Dyerberg J, Selleck M, Stender S (2008) Nutrition transition and its relationship to the development of obesity and related chronic diseases. Obes Rev 9(Suppl 1): 48-52.

19. Deurenberg P, Yap M, Staveren WA (1998) Body mass index and percent body fat: A meta analysis among different ethnic groups. Int J Obesity 22(12): 1164-1171.

20. Christopher LD, Mark WR, Siet M (2001) Livestock to 2020: the revolution continues. International Trade in Livestock Products Symposium.

21. Christopher LD, Mark WR, Siet M (2001) Livestock to 2020: the revolution continues. Iternational Agricultural Trade Research Consortium (IATRC).

22. Noor MI (2002) The nutrition and health transition in Malaysia. Public Health Nutr 5(1A): 191-195.

23. Kosulwat V (2002) The nutrition and health transition in Thailand Public Health Nutr 5(1A): 183-189.

24. Popkin BM (2000) Urbanization and the Nutrition Transition. Focus 3 , Brief 7 of 10 .

25. Chantal B, Mickey C, Rolph H (2009) Trade and social determinants of health. The Lancet 373(9662): 502-507.

26. Josef S, Prakash S (2005) The nutrition transition to 2030. Why developing countries are likely to bear the major burden. Food Economics - Acta Agriculturae Scandinavica, Section C, 2(3-4): 150166.

27. Kim S, Moon S, Popkin BM (2000) The nutrition transition in South Korea. Am J Clin Nutr 71(1): 44-53.

28. Popkin BM, Horton S, Kim S (2001) The Nutritional Transition and Diet-Related Chronic Diseases in Asia. Implications for Prevention.

29. Amuna P, Zotor FB (2008) Epidemiological and nutrition transition in developing countries: impact on human health and development. Proc Nutr Soc 67(1): 82-90. 